\title{
Reduction of NAA/Cr ratio in a patient with reversible posterior leukoencephalopathy syndrome using MR spectroscopy
}

Jun-Liang Yuan ${ }^{1}$, Shuang-Kun Wang ${ }^{2}$, Xiao-Juan Guo ${ }^{2}$, Ling-ling Ding ${ }^{1}$, Hua Gư ${ }^{2}$, Wen-Li Hu ${ }^{1}$

\author{
${ }^{1}$ Department of Neurology, Beijing Chaoyang Hospital, Capital Medical University, \\ Beijing, China \\ 2Department of Radiology, Beijing Chaoyang Hospital, Capital Medical University, \\ Beijing, China
}

Submitted: 10 July 2016

Accepted: 2 September 2016

Arch Med Sci Atheroscler Dis 2016; 1: e98-e100

DOI: 10.5114/amsad.2016.62376

Copyright $\odot 2016$ Termedia \& Banach

Reversible posterior leukoencephalopathy syndrome (RPLS) is a rare syndrome associated with hypertension, use of immunosuppressive medication and cytotoxic agents, which is characterized by clinical manifestations of headache, seizures, visual disturbances, confusion and other neurologic symptoms. The syndrome is usually reversible, but the mechanism of RPLS remains unclear [1]. Up to now, the most accepted theory is that rapidly elevated blood pressure (BP) exceeds the autoregulatory capability of the brain vasculature, which results in brain hyperperfusion and breakdown of the blood-brain barrier (BBB) with focal transudation of fluid and protein. The technique of proton MR spectroscopy (MRS) allow the in vivo measurement of various neurochemicals, which can provide biochemical information regarding the metabolism, cellular composition and pathophysiology of brain lesions. N-acetyl aspartate (NAA) is referred to as a neuronal marker. Decreased NAA indicates neuronal dysfunction that may be reversible. Choline (Cho) is referred to as a cell membrane and myelin marker. Creatine ( $\mathrm{Cr}$ ), as a marker of energy metabolism, is mainly used as an internal reference to assess the other two metabolites. To our best knowledge, only isolated reports of MRS have been published about RPLS. We herein report our findings of quantitative, multi-voxel MRS in a patient with RPLS.

A 39-year-old man was admitted to the emergency room of Beijing Chaoyang Hospital with the symptoms of headache, dizziness, nausea and vomiting for about 4 weeks. He had a 3-year history of hypertension but did not take any antihypertensive drugs, 6 years history of gout, and a history of smoking and drinking for 20 years. He had a family history of hypertension and ischemic stroke. The ambulatory BP monitoring showed that his BP initially fluctuated from $130 \mathrm{~mm} \mathrm{Hg}$ to $200 \mathrm{~mm} \mathrm{Hg}$ for systolic BP and from $96 \mathrm{~mm} \mathrm{Hg}$ to $149 \mathrm{~mm} \mathrm{Hg}$ for diastolic BP during $24 \mathrm{~h}$. Neurological physical examination revealed no abnormalities except for left positive Babinski sign. Physical examination, especially the cardiological examination, also was normal. The cognitive tests showed that the scores of the Mini-Mental State Examination and Montreal Cognitive Assessment were 30 .

The laboratory examinations including blood routine, urine routine, coagulation function, biochemistry, immune index, thyroid function, tumor biomarker, hormone levels (such as corticosterone, reproductive hor-

\author{
Corresponding author: \\ Dr. Wen-Li Hu \\ Department of Neurology \\ Beijing Chaoyang Hospital \\ Capital Medical University \\ Beijing 100020, China \\ Phone: +861085231398 \\ E-mail: huwenli@sina.com
}


mone), heavy metal testing (such as copper, lead, manganese in serum and urine), hepatitis virus, human immunodeficiency virus, and syphilis were normal. The uric acid was $486 \mu \mathrm{mol} / \mathrm{l}$, homocysteine $20 \mu \mathrm{mol} / \mathrm{l}$, high-sensitivity C-reactive protein (hs-CRP) $4.98 \mathrm{mg} / \mathrm{l}$. The cerebrospinal fluid (CSF) was clear and the pressure was $180 \mathrm{~mm} \mathrm{H}_{2} \mathrm{O}$. The total protein of CSF was $80 \mathrm{mg} / \mathrm{dl}$, glucose $3.06 \mathrm{mmol} / \mathrm{l}$, chloride $125.1 \mathrm{mmol} / \mathrm{l}$, white blood cell count $4 / \mathrm{mm}^{3}$. The genes of white matter changes (such as notch3, ABCD, PEX) were analyzed, and no abnormal genes were found. Cardiac ultrasonography, renal artery ultrasound, carotid Doppler, electrocardiography and electroencephalography were normal. Fundoscopy also revealed hypertensive retinopathy.

An emergent head computed tomography (CT) scan showed multiple low densities involving the bilateral parietal and frontal lobes (Figure 1). Brain magnetic resonance imaging (MRI) showed severe diffuse hyperintensities of white matter on the bilateral cerebral hemisphere (Figure $2 \mathrm{~A}-\mathrm{F}$ ), without gadolinium enhancement (Figure $2 \mathrm{G}-\mathrm{I}$ ). The MRS was obtained from the volume of interest positioned on the centrum semiovale. The ratio of NAA/Cr was 1.53, which was much lower than the left normal white matter with the ratio of NAA/ $\mathrm{Cr}$ 1.97. The ratio of $\mathrm{Cho} / \mathrm{Cr}$ was 1.20 , which was also lower than the left normal white matter with the ratio of $\mathrm{Cho} / \mathrm{Cr}$ 1.48. Computed tomography angiography, magnetic resonance angiography and magnetic resonance venography revealed no abnormalities.

The patient's BP returned to normal after treatment with combined antihypertensives within 1 week. Two weeks later, he recovered rapidly from

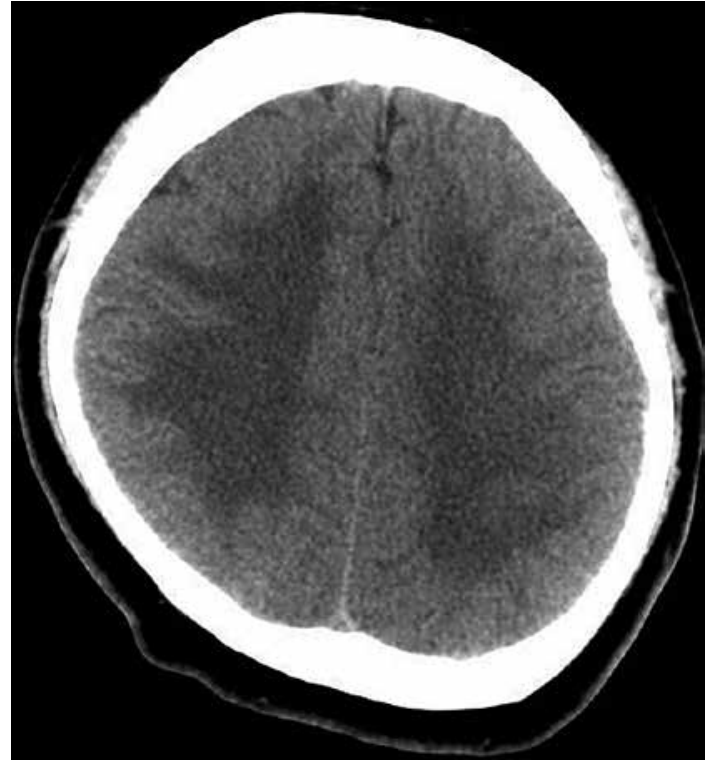

Figure 1. The emergent head computed tomography images showed that there were multiple low densities involving the bilateral parietal and frontal lobes

the above clinical symptoms and was discharged from our hospital. After 10 weeks' follow-up, another brain $\mathrm{CT}$ was performed, which showed the resolution of such low densities of the white matter (Figure 3). There was no relapse during 2 years of follow-up.

In our present case, according to the age, long history of severe hypertension, clinical features, subsequent improvement after control of BP, neuroimaging, and the follow-up manifestations, the presentation was strongly suggestive of RPLS. Especially, the diffusion-weighted imaging (DWI) sequence and ADC map also reflected the prepon-



Figure 2. Head MRI images with T1WI (A and F), T2WI (B), fluid-attenuated inversion recovery (FLAIR, C), diffusion-weighted imaging (DWI, D), apparent diffusion coefficient (ADC, E), gadolinium enhancement (G-I). Note high signal intensity in the bilateral cerebral hemisphere 




Figure 3. After 10 weeks' follow-up, another brain CT was performed, which showed the resolution of such low densities of the white matter

derant existence of vasogenic edema, other than cytotoxic edema in the involved white matter. The reduced $\mathrm{NAA} / \mathrm{Cr}$ ratio also played a crucial role in the diagnosis.

The RPLS is an acute neurological syndrome characterized by an abrupt and dramatic rise in $\mathrm{BP}$ associated with central nervous system signs, including headache, seizures, visual disturbances and altered mental status. The syndrome is usually reversible, and the prognosis is favorable after control of BP. The most common abnormalities are located in the parieto-occipital areas, consistent with vasogenic edema involvement. However, the underlying pathophysiology of RPLS is not well understood. Vasogenic edema may be one widely accepted mechanism. This hypothesis proposes that ischemia or toxic drugs result in arterial endothelial injury, temporary dysfunction of autoregulatory capabilities and damage of the BBB, then vasogenic edema occurs consequently [2, 3].

The technique of MRS may be helpful to distinguish RPLS from other focal lesions or encephalopathy, such as infarction, tumor, or demyelination, which may present with similar conventional MR imaging appearances. The MRS reveals the state of tissue metabolism by detecting the concentration of metabolites, and thus indicates the pathology of the lesions. To our best knowledge, the findings on RPLS using MRS are rare and inconsistent [4]. For example, Eichler et al. found that 2 patients with RPLS showed increased levels of both Cho and $\mathrm{Cr}$, and decreased NAA in both normal and abnormal regions using MRS [5]. The inconsistencies may be attributed to the variations in the times when MRS was performed, the location of the region of interest, and the severity of the disease.
In conclusion, proton MRS may be considered to be helpful in the diagnosis and investigation of the underlying pathophysiology of RPLS. Further studies, such as with serial follow-ups, using MRS and other techniques of multimodal MRI, are needed to understand the mechanisms underlying RPLS.

\section{Acknowledgments}

This work was supported by the National Natural Science Foundation of China (81301016) and Beijing Municipal Administration of Hospitals' Youth Program (QML20150303).

\section{Conflict of interest}

The authors declare no conflict of interest.

\section{References}

1. Zhang P, Li X, Li Y, et al. Reversible posterior leukoencephalopathy syndrome secondary to systemic-onset juvenile idiopathic arthritis: a case report and review of the literature. Biomed Rep 2015; 3: 55-8.

2. Yamada SM, Kitagawa R, Teramoto A. A case of reversible posterior leukoencephalopathy syndrome with acute hypotension. Neurol Sci 2011; 32: 165-8.

3. Schneider JP, Krohmer S, Gunther A, et al. Cerebral lesions in acute arterial hypertension: the characteristic MRI in hypertensive encephalopathy. Rofo 2006; 178 : 618-6.

4. Lee SY, Kim SH, Lee SH, et al. Serial MR spectroscopy in relapsing reversible posterior leukoencephalopathy syndrome. Neurologist 2009; 15: 338-41.

5. Eichler FS, Wang P, Wityk RJ, et al. Diffuse metabolic abnormalities in reversible posterior leukoencephalopathy syndrome. Am J Neuroradiol 2002; 23: 833-7. 